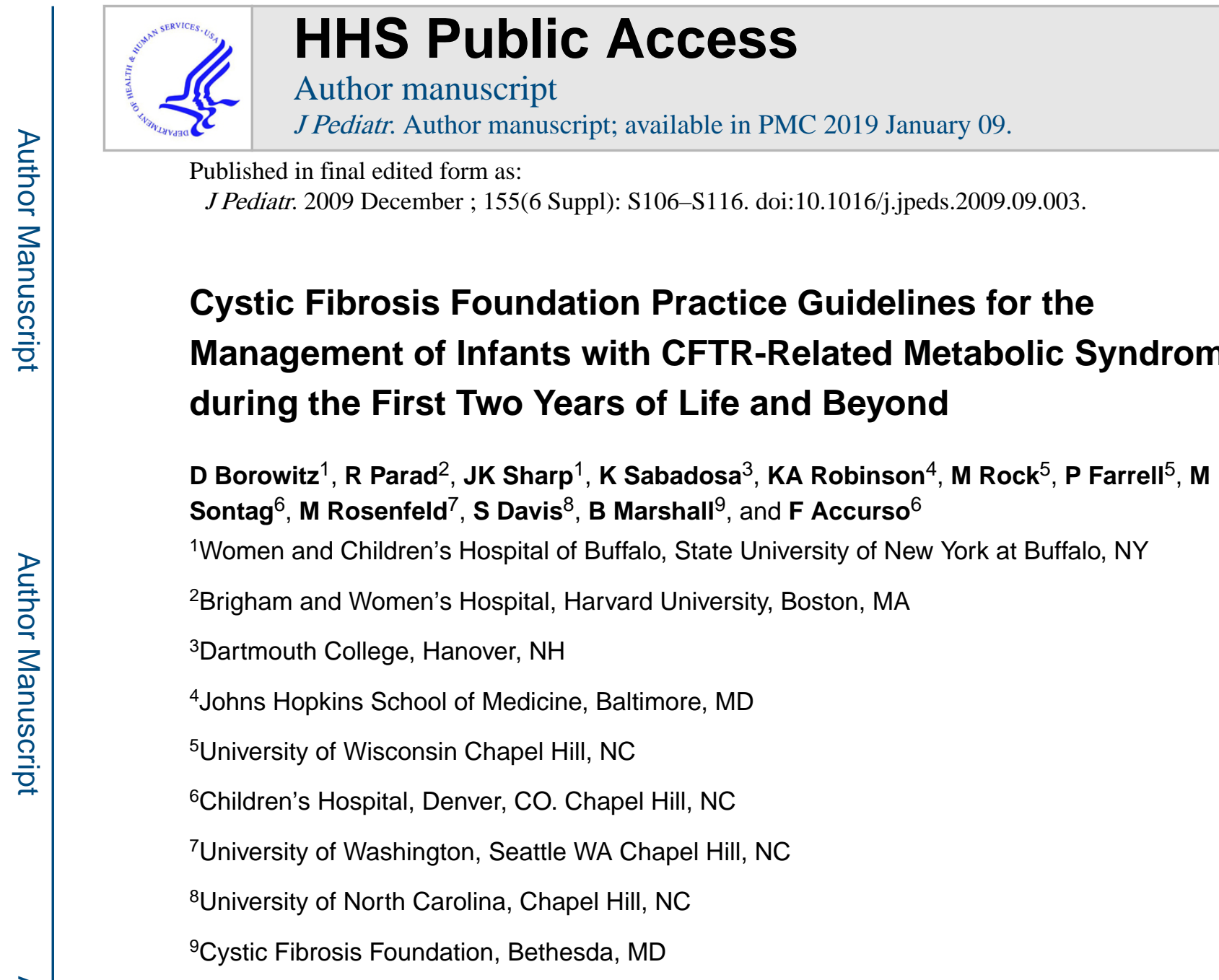

\title{
Abstract
}

Through early detection, newborn screening (NBS) for cystic fibrosis (CF) offers the opportunity for early intervention and improved outcomes. NBS programs screen for hypertrypsinogenemia and most also identify mutations in the CF transmembrane conductance regulator (CFTR) gene. Individuals identified by NBS are diagnosed with CF if they have an elevated sweat chloride or if they have inherited two disease-causing mutations in the CFTR gene. Mutations in the CFTR gene can cause CF, but not all CFTR mutations are disease-causing. The term CFTR-related metabolic syndrome (CRMS) is proposed to describe infants with hypertrypsinogenemia on NBS who have normal or intermediate sweat chloride values and 2 CFTR mutations, including at least one that is

Corresponding author: Bruce C. Marshall, MD, Cystic Fibrosis Foundation, 6931 Arlington Road, Bethesda, MD 20814. Contributors:

Frank Accurso, MD * (The Children's Hospital, Denver); Drucy Borowitz, MD (Women and Children's Hospital of Buffalo); Stephanie Davis, MD * (University of North Carolina at Chapel Hill); Henry L. Dorkin, MD * (Children's Hospital, Boston, MA);Ginny Drapeau, BSN,RN * (Connecticut Children's Medical Center); Philip Farrell, MD, PhD * (University of Wisconsin Medical School); Cindy Fortunato * (CF parent); Timothy Geleske, MD * (Northwestern University); Valerie Gilchrist, MD (University of Wisconsin);Christopher Harris, MD * (Vanderbilt University Medical Center); Melvin Heyman, MD * (University of California San Francisco); Michelle Howenstine, MD * (Riley Hospital for Children); Suzanne Michel, MPH, RD, LDN * (Children's Hospital of Philadelphia); Brian O'Sullivan, MD* (University of Massachusetts Medical Center); Richard Parad, MD, MPH * (New England Newborn Screening Program); George Retsch-Bogart, MD * (University of North Carolina at Chapel Hill); Karen A. Robinson, MSc (The Johns Hopkins University); Michael Rock, MD * (University of Wisconsin Medical School); Margaret Rosenfeld, MD, MPH (University of Washington, Seattle); Kathy Sabadosa, MPH (Dartmouth-Hitchcock Medical Center); Jack Sharp, MD * (Women and Children's Hospital of Buffalo); L. Terry Spencer, MD * (Children's Hospital, Boston MA); Lori Stark, $\mathrm{PhD}$ * (Children's Hospital Medical Center, Cincinnati); Terry White, PhD (Cystic Fibrosis Foundation); Preston W. Campbell III, MD (Cystic Fibrosis Foundation); Robert J. Beall, PhD (Cystic Fibrosis Foundation); Bruce Marshall, MD, PhD (Cystic Fibrosis Foundation); Leslie Hazle, RN (Cystic Fibrosis Foundation); Sarah Waybright (Cystic Fibrosis Foundation)

* participated in survey 
not known to cause $\mathrm{CF}$, and thus do not meet $\mathrm{CF}$ Foundation guidelines for the diagnosis of $\mathrm{CF}$. Individuals with one CF-causing mutation, no mutation on the other allele and an intermediate sweat chloride are presumed to be CF carriers. With what is now near-universal CF NBS in the United States, an increasing number of infants with CRMS are being identified. Given our inadequate knowledge of the natural history of CRMS, standards for diagnosis, monitoring and treatment are absent. This document aims to help guide the monitoring and care of individuals with CRMS while our knowledge base on appropriate management evolves.

\section{Introduction and Definition}

The Cystic Fibrosis Foundation convened a group of experts in July 2007 to create guidelines for the management of infants with cystic fibrosis (CF), many of whom are diagnosed by newborn screening (NBS) (1) The National Newborn Screening and Genetics Resource Center in Austin, Texas reports that over 90\% of newborns in the United States undergo CF NBS. NBS programs in the US identify infants as being at risk for CF if they have hypertrypsinogenemia, and most also identify mutations in the CF transmembrane conductance regulator (CFTR) gene. Infants identified by NBS are diagnosed with CF if they have an elevated sweat chloride value or if they have inherited two disease-causing mutations in the CFTR gene in an autosomal recessive fashion. Some mutations in the CFTR gene can cause CF, but not all CFTR mutations cause CF A CF Foundation Consensus Report on Guidelines for Diagnosis of CF has recently been published which sets cutoff values for the quantitative pilocarpine iontophoresis test for sweat chloride (2), and a recent international group has proposed a categorization scheme for some commonly encountered CFTR mutations: "CF-causing mutations", "mutations associated with CFTR-related disorder", "mutations with no clinical consequences", and "mutations of unproven or uncertain clinical relevance" $(3)^{1}$ (Table 1). Infants without a diagnostic sweat chloride value $\geq 60 \mathrm{mmol} / \mathrm{L}$ or without two CF-causing mutations present the clinician and family with a diagnostic dilemma (Figure 1). Older individuals in these categories have been called "atypical CF", "non-classical CF", "CFTR-related disorders", "low risk genotype" or "mild variant CF", but they present for diagnostic evaluation because of signs or symptoms, whereas infants identified by CF NBS are asymptomatic. The Guidelines for Diagnosis of CF in Infants through Older Adults labels infants in this uncertain category as "possible CF" or "CF very unlikely" (2). For infants identified by CF NBS programs, we propose the designation CFTR-related metabolic syndrome (CRMS) to provide a clear name to families that:

1. does not imply that the infant has $\mathrm{CF}$

2. includes patients with mutations that are of unproven or uncertain clinical relevance in addition to those associated with CFTR-related disorder, and

3. can be associated with an International Classification of Disease (ICD) code (277.9)

We sought to define the issues confronting CF caregivers and families when an infant is found to have CRMS. What follows is based on a consensus of opinions from experts in the field using the Delphi methodology to refine the final statements (see Methods). These 
suggestions should be taken in the context of a rapidly expanding evidence base. The central difficulty in writing these guidelines is that some clinicians hesitate to apply a medical label to infants who may never develop any symptoms. Others have experience with patients who have developed significant symptoms and these physicians are reluctant to miss an opportunity for preventive care. It is recognized that our knowledge of genotype-phenotype association is evolving and that our data on the natural history of this syndrome currently are limited. The development of a wide spectrum of phenotypes has been observed in those with CRMS; however we do not yet know how to predict which individuals are at risk of becoming symptomatic and the severity of symptoms in those affected. In some patients, signs and symptoms may ultimately lead to a diagnosis of CF. Others will develop a phenotype consistent with CFTR-related metabolic syndrome (e.g. isolated pancreatitis or CBAVD). Still others will never develop symptoms. It is for this reason that the following monitoring and treatment guidelines have been developed. In cases where evolving phenotypic features develop or repeat sweat chloride values are $\geq 60 \mathrm{mmol} / \mathrm{L}$ so that a diagnosis of CF is made, care guidelines developed for patients with CF should be used.

\section{Methods:}

Since most of our understanding of CRMS is based on clinical case series, we recognized that evidence-based guidelines would not be possible. We wanted to avoid use of a nonsystematic, opinion-driven approach. (4). We chose the Delphi methodology to prospectively and formally define how we would determine consensus or lack thereof with the proposed recommendations (5). The CF Foundation charged a small working group with creating a general framework for management of CRMS based on knowledge and experience as well as on the recommendations from the CF Foundation Consensus Report on Guidelines for Diagnosis of CF (2). The output of that group included a series of proposed care statements. The statements were then circulated to a larger panel of experts which included some members of the group that developed Guidelines for Management of Infants with CF (1) and the CF Center Directors in Massachusetts, a state with five CF Care Centers and nine years of experience with CF NBS were also included for a total of 18 participants (see designated participants listed at the end of the manuscript). For each statement, participants were asked to rate their agreement with the statement on a scale of 0 to 9 , where 0 is complete disagreement and 9 is complete agreement. Participants also were given an opportunity to provide additional information along with their score for each statement, i.e., suggest rewording or present an argument or literature in support of the score. The results were tabulated: values between 7 and 9 were considered good agreement; consensus was defined as $>=80 \%$ participants in good agreement.

Revisions were made to the statements in anticipation of a second round of input from the experts. In some instances, statements that directly contradicted each other were presented in the initial round of statements. In these cases, statements were dropped in Round 2 if an opposing statement achieved consensus in Round 1.Questions with $<80 \%$ agreement were explored further and were reworded for a second round of the survey based on comments. For example, in some questions the interval for intervention was changed from 1 week to 2 weeks. In others, the wording was changed to emphasize intervention only if individuals were symptomatic or had a test result that informed the action. 
In Round 2, the initial statements were sent out to the working group with a summary of results (tabulated scores including mean, median and range, and summarized comments) to allow for change in response. New or revised statements were also circulated and participants were asked for rating of level of agreement. Results are in Table 2. Statements for which consensus was achieved are also listed throughout the text at the end of each relevant section.

A draft of the guidelines was posted on a secure website for comment from CF Center care teams (physicians and ancillary care providers) and was revised as appropriate. Wording for two recommendations was modified in a manner that improved the recommendation but did not change the meaning of the original statement for which consensus was achieved. These changes are noted in Table 2.

\section{Diagnosing CFTR-Related Metabolic Syndrome:}

The CF Foundation Consensus Report on Guidelines for Diagnosis of CF describes the early diagnostic process and the reader is referred to the pertinent Figure (2). This Consensus Report recommends sweat chloride testing and follow-up at 6 to 12 month intervals until the diagnosis is clear. As opposed to older individuals, infants are unlikely to have symptoms that would help clarify the diagnosis. Thus, infants may be labeled as having CRMS if the diagnosis of CF cannot be definitively made or ruled out, and these Guidelines address those individuals.

In most cases infants found to have hypertrypsinogenemia on NBS undergo an initial sweat test by 2- 4 weeks of age and most will also have mutation testing. If the initial sweat test and mutation testing (if available) is non-diagnostic, a repeat sweat test should be performed to confirm the intermediate value or demonstrate resolution into the normal $(<30 \mathrm{mmol} / \mathrm{L})$ or abnormal ( $\geq 60 \mathrm{mmol} / \mathrm{L}$ ) range. Sweat testing should be performed at an accredited CF Center. The CF Foundation Consensus Report on Guidelines for Diagnosis of CF (2) includes an algorithm that recommends a repeat sweat test for such individuals with an intermediate result on the first test between two and six months of age. The Massachusetts CF NBS program presented data at the North American Cystic Fibrosis Conference in 2007 from 102 infants with a positive newborn screen for CF (with 0,1 or 2 CFTR mutations identified) and an intermediate sweat chloride value. Of these infants, 72 had a repeat sweat chloride measurement. The sweat chloride concentration in 38 infants (about half of those undergoing repeat testing) was $<30 \mathrm{mmol} / \mathrm{L}$ after a median interval of 25 days from the first test (median age at second test was 62 days). Thus, repeating the sweat test at two months of age may resolve the diagnosis in many instances. Of note, infants with a normal sweat chloride concentration may still have CRMS if two CFTR mutations have been identified (Figure 1).

The CF Foundation Consensus Report on Guidelines for Diagnosis of CF recommends that infants with sweat chloride values persistently in the intermediate range should undergo an extensive CFTR DNA analysis (2). The mutation analysis ordered should be of a higher investigatory level than the mutation panel that was used in the initial newborn screen. For example, in the Massachusetts CRMS follow-up algorithm, gene scanning or gene 
sequencing along with insertion and deletion analysis is recommended at this stage. Some geneticists recommend gene sequencing as opposed to gene scanning for more definitive diagnosis. The repeat sweat test and genotyping are best performed at a CF Center where an initial evaluation by a CF clinician can be arranged and where genetic counseling is available. The results from the genetic analysis will enable clinicians to establish a diagnosis of CF if two CF-causing mutations are documented. Infants with two CFTR mutations that are not both clearly disease-causing and a persistently intermediate sweat chloride concentration should have a third sweat test at about six months of age. Frequent, repeated sweat tests between two and six months of age are unlikely to add information and may further confuse the situation. Thus, after complete assessment, healthy hypertrypsinogenemic newborns should be considered to have CRMS if they have either:

- $\quad$ intermediate sweat chloride concentrations on at least 2 occasions and less than two identified disease-causing CFTR mutations OR

- a normal sweat chloride and two identified CFTR mutations, of which no more than one is known to be disease-causing

Infants with CRMS should be monitored because they are at increased risk for developing CF-like symptoms. Furthermore, in some individuals, evolving signs and symptoms, new information about disease-causing CFTR mutations, or change in sweat chloride concentrations may ultimately lead to a diagnosis of CF. To be consistent with the ICD-9 coding for $\mathrm{CF}$ diagnoses, which are in the category of metabolic disorders, and because by definition all infants will have hypertrysinogenemia, the recommended ICD-9 code for individuals with CRMS is 277.9 (unspecified disorder of metabolism).

\section{Discussing the Implications of a CFTR-Related Metabolic Syndrome:}

It can be very difficult to explain the implications of CRMS to families who have a healthyappearing infant. Extensive mutation analysis can define a diagnosis, but it also can be confounding when complex alleles are found. Some examples are:

- $\quad$ CFTR mutations with variable penetrance: CF NBS programs have reported that up to $7 \%$ of infants screened using a trypsinogen/DNA multi-mutation algorithm in which two mutations are identified will have one CF-causing mutation and R117H-T7 (6). Some individuals with a CF-causing mutation on one allele and $\mathrm{R} 117 \mathrm{H}$ associated with the T7 intron-8 polythymidine sequence (IVS8) on the other allele may develop CF symptoms, while others will not, though symptoms are rarely seen in early childhood and may not develop until adulthood (7) (8). Some CFTR mutations, including D1152H, have widely variable phenotypes (9). If mutations such as $\mathrm{D} 1152 \mathrm{H}$ are found in trans with other phenotypically variable CFTR mutations, such as R117H-T7, an already confounded picture becomes more complicated.

- Intronic mutations and intron-intron mutation interactions: The T5 IVS-8 allele may result in an abnormal phenotype if it is in trans with a CF-causing mutation such as F508del (10). Analysis for thymidine-guanine repeat number adjacent to T5 may influence penetrance (11) (12). When TG12-T5 or TG13-T5 is found in 
compound heterozygosity with a CF-causing mutation, it may result in CFTRrelated syndrome such as absence of the vas deferens or idiopathic pancreatitis, or may even lead to lung disease consistent with a diagnosis of CF (10).

- $\quad$ Non-CFTR mutations that affect sweat chloride: Non-CFTR genes may cause increased sweat chloride, for example, mutations in the sodium channel (SCNN1B). Genetic testing for SCNN1B mutations is commercially available (http://www.hopkinsmedicine.org/dnadiagnostic/CFlikeBENaC.htm ).

- $\quad$ CFTR sequence variants with unknown or uncertain clinical relevance: Many "private" or rarely identified sequence variants that lead to missense mutations are of unknown or uncertain clinical significance, but possibly could act either as a clinically significant mutation when found in trans to a disease-causing CFTR mutation, could have additive effects when found in cis with other mutations that have not been proven to cause disease, or may be benign markers that cosegregate with a disease causing mutation.

These scenarios reinforce the fact that it is difficult to predict outcomes based on genotype, but in a world where gene sequencing is available, CF specialists need to be informed and prepared to discuss these difficult problems with families. The authors of the Consensus on Use and Interpretation of CF Mutation Analysis in Clinical Practice recognized these limitations of genetic diagnosis and stated that phenotype is more important than genotype (3). Table 3 lists the most important issues to be discussed with the family at the time of initial discussion of the diagnosis. Parent-friendly teaching materials are in Appendix 1. These list symptoms that should prompt the family to visit their primary care provider (PCP). A genetic counselor should be present at the discussion of CRMS, if possible, and should be available to the family in the future.

- The CF Foundation recommends that for individuals with CRMS, a genetic counselor should be present, if possible, in the discussion of genetic findings (83\% agreement)

\section{Coordination of Care with Primary Care Providers:}

Families will be making more visits to their PCP than to their CF specialist during the first two years of life, especially during the first six months of life when the family may be awaiting genetic testing results and the infant is not old enough for a third sweat test. The CF specialist should communicate directly with the PCP to explain the implications of CRMS immediately following the initial assessment by the specialist, when the infant is approximately 1-2 months of age. The PCP should contact the CF specialist if an infant with CRMS is not gaining weight, has loose stools and/or flatus, has abdominal pain or has respiratory symptoms such as cough or wheezing that do not resolve in 2 weeks. Open communication with the $\mathrm{CF}$ specialist is important if there are concerning findings at any time, especially if they are severe or if they progress rapidly. The management of infants with CRMS relies on collaborative decision making between the PCP and CF specialist, and the role of the PCP is crucial, especially if there are geographic, financial, or other barriers to follow-up at the CF Center. These principles continue to be true throughout the 
individual's entire life, as symptoms may not develop until after the first few years of life. Key issues for PCPs are listed in Table 4.

O The CF Foundation recommends that for individuals with CRMS, the CF specialist should communicate directly with the primary care provider to explain the implications of CRMS (94\% agreement)

O The CF Foundation recommends that for individuals with CRMS, the primary care provider should contact the $\mathrm{CF}$ specialist if the infant is not gaining weight, has loose stools and/or flatus, has abdominal pain or has respiratory symptoms such as cough or wheezing that do not resolve in 2 weeks ((94\% agreement)

\section{Clinical Care:}

\section{The initial CF specialist assessment:}

In patients with a definitive $\mathrm{CF}$ diagnosis, clinical manifestations can occur in the first few weeks of life (13) (14). Although it is unlikely that symptoms will develop early in infants with CRMS, early symptoms have been reported (15). An infant with CRMS should have a clinical assessment by a CF specialist by no later than two months of age to allay anxiety and to establish a baseline. To prevent possible cross-infection, infants with CRMS should be assessed in a clinic adherent to CF Foundation guidelines for CF patients (16), or in a separate clinic area from CF patients as local conditions allow. Care may be provided by a CF specialist alone, outside the context of a full CF team. The CF Center nurse or social worker may need to be involved to respond to telephone contacts or families who are having difficulty coping with the uncertainty of diagnosis. Other members of the CF care team do not need to see every individual with CRMS; this can be scheduled on an as-needed basis. Care in the context of a full CF Center may have the unintended consequence of reinforcing that the child has $\mathrm{CF}$, when in fact that is not the diagnosis.

O The CF Foundation recommends that for individuals with CRMS, a clinical assessment by a CF specialist should be performed by 2 months of age ( $94 \%$ agreement)

The CF Foundation recommends that, to prevent possible cross-infection, infants with CRMS should be assessed in a clinic adherent to CF Foundation guidelines suggested for CF patients, or in a separate clinic area from CF patients as local conditions allow (83\% agreement)

O The CF Foundation recommends that for individuals with CRMS, CF Center ancillary personnel may need to see individuals with CRMS, this can be scheduled on an as-needed basis (100\% agreement)

The following should be done at the time of the initial assessment: comprehensive history including family history, and physical examination including accurate weight, length and head circumference measurements, an objective measure of pancreatic function, and an oropharyngeal culture (processed as a CF culture). A chest x-ray should be obtained only if respiratory symptoms are present. The expert panel was queried regarding whether the following laboratory tests may be considered at the time of the initial assessment: complete 
blood count, liver function tests, albumin, vitamin A and vitamin E levels; consensus was not achieved. A recent statement by the European Cystic Fibrosis Society Neonatal Screening Working Group (17) suggests an extensive evaluation of infants in this category, and some Centers in the US and Canada have specific diagnostic algorithms to attempt to better classify infants with CRMS. However, routine use at all CF Centers of more extensive testing such as infant pulmonary function testing, chest computed tomography scanning (CT), bronchoalveolar lavage, nasal potential difference measurements and /or rectal ion channel and potential difference measurements is not recommended.

$\mathrm{T}$ The CF Foundation recommends that for individuals with CRMS, the following should be done at the time of the initial assessment: comprehensive history and physical including accurate weight and height measurements, an objective measure of pancreatic function, and an oropharyngeal culture (94\% agreement)

The CF Foundation recommends that for individuals with CRMS, a chest X-ray should be obtained if respiratory symptoms are present (94\% agreement)

Persistent infection of the airways with Pseudomonas aeruginosa has been considered to be one of the phenotypic features consistent with a diagnosis of CF (2). In one report, oropharyngeal swabs positive for Pseudomonas were seen intermittently in a majority of infants with an uncertain diagnosis (18). However, Pseudomonas aeruginosa can be seen in healthy infants. In one study, 20 healthy infants had oropharyngeal swabs obtained every three months during the first year of life (20). Non-mucoid Pseudomonas aeruginosa grew in one infant in the 6 month cohort and two twin infants who were living in the same household in the 12 month cohort. Furthermore, respiratory syncitial virus (RSV) is a frequent cause of respiratory symptoms in infants, and RSV has been found to mediate Pseudomonas binding to normal epithelial cells (19). However, the presence of this organism in an infant with an elevated though not diagnostic sweat test warrants concern, and treatment of patients per CF protocol may be considered if Pseudomonas aeruginosa is found on oropharyngeal swab (1). Although bronchoscopy should be performed in infants with CRMS only for a clear-cut clinical indication, the presence of Pseudomonas in the lower respiratory tract would be strongly suggestive of CF. Staphylococcus aureus or Hemophilus influenzae should not be treated routinely in patients with CF (1), and by inference, should not be treated in individuals with CRMS.

- $\quad$ The CF Foundation recommends that individuals with CRMS may be treated per CF protocol if Pseudomonas aeruginosa is found on oropharyngeal swab, even if a definitive diagnosis of CF has not been established ( $83 \%$ agreement)

\section{Subsequent CF Specialist Assessments:}

The sweat chloride test should be repeated at 6 months of age. An increase in the sweat chloride value $\geq 60 \mathrm{mmol} / \mathrm{L}$ establishes a diagnosis of $\mathrm{CF}$. A sweat chloride value $<40$ $\mathrm{mmol} / \mathrm{L}$ after 6 months of age is generally not consistent with a diagnosis of CF, although $\mathrm{CF}$ has been reported in rare instances in patients with an intermediate sweat chloride value (2). If the sweat chloride value remains in an intermediate range (40-59 $\mathrm{mmol} / \mathrm{L}$ for individuals 6 months or older) in an asymptomatic infant who does not have two CF-causing mutations, a diagnosis of CF is possible but should not be made unless published $\mathrm{CF}$ 
Foundation criteria are met. Asymptomatic infants with CRMS should be seen by a CF specialist at least twice during the first year of life and once a year thereafter. Some asymptomatic infants may need more visits, depending on the comfort level of the family. Suboptimal weight gain or recurrent respiratory symptoms do not make the diagnosis of $\mathrm{CF}$ certain, but in these instances, more frequent follow-up in the context of a CF Center care may be warranted. Although in some individuals symptoms may not appear until adulthood if at all, at present it is impossible to predict which individuals will need intervention. Thus, ongoing surveillance is recommended. Oropharyngeal swabs that are processed in the same manner as CF sputum should be obtained at each visit (Figure 2).

O The CF Foundation recommends that individuals with CRMS who are asymptomatic should be seen at least twice during the first year of life and once a year thereafter. Some asymptomatic individuals may need more visits, depending on the comfort level of the family (94\% agreement)

O The CF Foundation recommends that for individuals with CRMS, oropharyngeal cultures should be obtained at every visit (94\% agreement)

\section{Initial Therapy:}

Definitive recommendations for preventive therapies for infants with CRMS are difficult to make. Infants with $\mathrm{CF}$ are at risk for salt depletion if they do not receive supplemental salt. They are further at risk if they become ill, including fever, vomiting and/or diarrhea and when exposed to high environmental temperatures, including warm homes and overbundling, or living in a hot, arid climate. Although the extent of salt loss is not likely to be as great in infants with CRMS as in infants with CF, the amount of salt in the sweat for those with sweat chloride values in the intermediate range is by definition greater than three standard deviations above the norm. Members of the expert panel were queried concerning salt supplementation for infants with CRMS but consensus was not achieved. Patients with $\mathrm{CF}$ can be deficient in the fat-soluble vitamins A, E, D and K. Individuals with CRMS are almost universally pancreatic sufficient and are thus at low risk for vitamin deficiency. Members of the expert panel did not achieve consensus on a recommendation to check fatsoluble vitamin levels. Some clinicians may choose to measure fat-soluble vitamin levels, and if they are found to be low the expert panel recommended supplementation with vitamins designed for patients with $\mathrm{CF}$ as opposed to standard multivitamins. Of note, further diagnostic testing also should be pursued to evaluate pancreatic, intestinal or hepatic causes of malabsorption in patients with fat-soluble vitamin deficiency.

While the CFF cannot recommend routine measurement of fat-soluble vitamin levels for individuals with CRMS, if laboratory evidence of a deficiency is documented, further evaluation for malabsorption is needed and multivitamins designed for people with CF should be prescribed (94\% agreement)

Because lung disease is the most serious complication of $\mathrm{CF}$ and has been described in individuals with CRMS, therapies to prevent lung disease were considered. The CF Foundation Evidence-Based Guidelines for Management of Infants with CF states, "evidence to support guidelines for early prevention or treatment of pulmonary disease in infants with CF is sparse" (1). Since it is unclear whether infants with CRMS will ever 
develop CF-like pulmonary complications, in the absence of clinical or radiologic evidence of lung disease, routine airway clearance therapy should not be prescribed. As is true for all children, individuals with CRMS should not be exposed to cigarette smoke. As per the American Academy of Pediatrics recommendation for all children, individuals with CRMS who are over 6 months of age should receive annual influenza vaccine, as well as all other routine immunizations.

T The CF Foundation recommends that for individuals with CRMS, in the absence of clinical or radiologic evidence of lung disease, routine airway clearance therapy should not be prescribed (94\% agreement)

O The CF Foundation recommends that individuals with CRMS should not be exposed to cigarette smoke (100\% agreement)

O The CF Foundation recommends that individuals with CRMS should receive annual influenza vaccine (94\% agreement)

\section{Subsequent Therapy:}

Patients with CF who are pancreatic sufficient (PS) have enough pancreatic enzyme secretion to prevent steatorrhea, but they do not have a normal pancreas. The amount of secretin-stimulated high-volume, bicarbonate-rich pancreatic fluid is diminished (21), so CF-PS patients may become PI and also are at risk for recurrent pancreatitis. Individuals with CRMS may have similar diminished pancreatic function. An objective measure of pancreatic functional status such as fecal elastase should be re-checked after the initial visit if there is poor weight gain, loose stools and/or excessive flatus, and/or abdominal pain. It should be noted that enteropathy can cause secondary pancreatic insufficiency and a low fecal elastase (22), thus infants with failure to thrive and low elastase should have the fecal elastase re-checked when their nutritional status improves. Patients with CF who are PS may have constipation as a result of loss of intraluminal intestinal fluid volume; constipation may contribute to a poor appetite and poor weight gain which could be misinterpreted as resulting from a change from pancreatic sufficiency to insufficiency. An abdominal x-ray should be considered in pancreatic sufficient individuals with CRMS with poor weight gain, loose stools and/or flatus, and/or abdominal pain to rule out constipation.

O The CF Foundation recommends that for individuals with CRMS, those who are pancreatic sufficient and have poor weight gain, loose stools and/or flatus, and/or abdominal pain should have an objective measure of pancreatic functional status to rule out conversion to pancreatic insufficiency (100\% agreement)

O The CF Foundation recommends that for individuals with CRMS, an abdominal $\mathrm{x}$-ray should be considered in those with poor weight gain, loose stools and/or flatus, and/or abdominal pain to rule out constipation ( $82 \%$ agreement)

As noted above, some individuals may develop respiratory complications while others will not. Viral illnesses are very common in infants and young children, so it may be difficult to determine if cough or other respiratory symptoms are within normal limits or related to the underlying CRMS. Individuals with cough that does not resolve after 2 weeks should be 
evaluated by their PCP (see recommendation in "Coordination with Primary Care Providers" section above). Airway clearance therapy may be considered if patients have recurrent or prolonged respiratory symptoms or have chest x-ray findings suggestive of lower airway disease. These infants will likely need a more in-depth diagnostic evaluation; an approach to the infant with CF and increased pulmonary symptoms has been described (1) and should be considered in infants with CRMS if increased respiratory symptoms persist to the point that airway clearance therapy is instituted. Counseling for the avoidance of smoke exposure should be incorporated into CF Center monitoring visits, and should continue to be reinforced by the PCP (see recommendation in "Initial Therapy" section above).

The CF Foundation recommends that for individuals with CRMS, airway clearance therapy may be considered if individuals have recurrent or prolonged respiratory symptoms or have chest $\mathrm{x}$-ray findings suggestive of lower airway disease (88\% agreement)

Males with CRMS who have the R117H mutation are likely to have absence of the vas deferens. The reproductive potential of males with CRMS with other mutations is unclear, however many men with obstructive azoospermia have abnormalities in a range of CFTR exons and/or introns (23) (24). Families need to be made aware of the potential for reproductive problems in males with CRMS, and at the appropriate age their sons should be offered testing to define their reproductive status. Physicians discussing CRMS should clarify that sexual function is normal even when reproductive potential is not.

\section{Future Research:}

Clinicians need evidence to determine the optimal clinical management of individuals with hypertrypsinogenemia, non-diagnostic sweat chloride values and/or ambiguous genotype, especially with the increase in these findings resulting from CF NBS. Given the uncertainty in the clinical outcome for individuals with CRMS, it is unclear how closely these individuals should be followed. Gathering clinical and genetic information through a central reporting system such as the CF Foundation Patient Registry would be an important first step to help us learn more about CRMS. Ideally, the development of new biomarkers as well as identification of genetic modifiers may enable us to provide more accurate prognostic information to families. These markers could also help determine which individuals with CRMS are at highest risk for complications and merit closer surveillance.

\section{Conclusion}

When CF NBS identifies an infant with CRMS, a complex and potentially difficult situation is created for the family, CF specialist and PCP. Care should be given to first, do no harm by creating a vulnerable child in the parents' eyes, since we believe that many of these individuals will live a long and healthy life with a low risk of developing signs or symptoms of CF. However, because some will become symptomatic and preventive care is preferable to symptomatic care, these individuals should not be lost to specialist follow-up. Newer techniques for diagnosis and prognosis may become available, and these should be offered to families. This document cannot encompass every situation and is meant to provide general recommendations rather than a set of rigid guidelines. By tracking the clinical 
outcomes of individuals with CRMS and through the development of new biomarkers as well as identification of genetic modifiers, we hope to alleviate the anxiety caused by this ambiguous situation.

\title{
Acknowledgments
}

\begin{abstract}
We are grateful to Dr. Wylie Burke, Professor and Chair of the Department of Medical History and Ethics at the University of Washington, Principal Investigator of the University of Washington Center for Genomics and Healthcare Equality (NIH-funded Center of Excellence in Ethical, Legal, and Social Implications Research) for her thoughtful comments.

We also appreciate the careful review of this manuscript by our colleagues Dr. Peter Durie, Senior Scientist, Program in Physiology and Experimental Medicine The Research Institute, Division of Gastroenterology, Hepatology and Nutrition Department of Pediatrics The Hospital for Sick Children Professor, Department of Pediatrics The University of Toronto and Dr. Tanya Gonska and Dr. Keith Ooi Research Fellows in the Program in Physiology and Experimental Medicine, The Research Institute, and the Division of Gastroenterology, Hepatology and Nutrition at the Hospital for Sick Children, Toronto and the Department of Pediatrics at the University of Toronto
\end{abstract}

\section{Appendix 1:: Family-friendly written explanation of CRMS CFTR-Related Metabolic Syndrome (CRMS)}

(CFTR stands for Cystic Fibrosis Transmembrane Regulator protein, which is the salt channel that the "CF gene" makes; see "What Causes CF")

Your child has "CFTR-Related Metabolic Syndrome (CRMS). Your infant had a newborn screen test for cystic fibrosis that gave an intermediate result. Your child does not have cystic fibrosis, but there are several reasons why we want your child to have regular check-ups with a doctor who is a cystic fibrosis specialist.

\section{What is CRMS?}

We say that a child has CFTR-Related Metabolic Syndrome (CRMS) when they have had a sweat test or a genetic test that gives an intermediate result. Sweat tests are used to make the diagnosis of cystic fibrosis (CF), a genetic (inherited) disease. CF causes thick mucus to get stuck in the breathing tubes or sinuses, the intestines and organs like the pancreas that are connected to the intestines, or the reproductive tract. Your child does not have CF, but one of two things makes us say that he or she has CRMS:

- The amount of salt in your child's sweat is higher than most children, although it is not high enough for us to say that he or she has CF. This could mean that your child is at higher risk to have problems in the breathing tubes or sinuses, the intestines and organs like the pancreas that are connected to the intestines, or the reproductive tract.

- Your child has one or two mutations in his or her CF genes that don't cause CF, but could mean that your child is at higher risk to have problems in the breathing tubes or sinuses, the intestines and organs like the pancreas that are connected to the intestines, or the reproductive tract. 


\section{What causes cystic fibrosis?}

To understand CRMS you need to know something about cystic fibrosis (CF). CF is a genetic (inherited) disease. Genes are what tells our body things like "you will have blue eyes" or "you will have curly hair". Genes comes in pairs. You get one from your mother and one from your father. When there is a change in the code in a gene it's called a mutation. Some mutations don't cause any problems at all, but some can cause diseases like CF. People with CF have a disease-causing mutation in each of their two CFTR genes, so they inherited one mutation from their mother and one mutation from their father.

\section{What are the symptoms of cystic fibrosis?}

The CFTR gene controls the salt channels in skin, and because they have mutations that cause disease, people with CF have very salty skin. The CF gene also controls the salt channels in parts of the body that are lined with tissues that are like skin - the breathing tubes and sinuses, the intestines and organs like the pancreas that are connected to the intestines, and the reproductive tract. People with CF get thick and sticky mucus in these parts of the body. The breathing tubes get clogged with thick mucus and often people get a germ called Pseudomonas (pronounced "soo-dah-MOAN-us"). When we find Pseudomonas, we treat it because people who have this germ can have worse lung function. People with CF can get very serious and permanent lung problems.

\section{What are the symptoms of CRMS?}

Your child is likely to remain healthy.-Some people with CRMS have developed problems in the breathing tubes or sinuses, the intestines and organs like the pancreas that are connected to the intestines, or the reproductive tract, but we don't know how many people with CRMS don't develop these problems. We think that the best thing to do is for your child to have regular check-ups with a CF specialist so that we can find and treat any early changes if they happen.

You should see your regular doctor and possibly your CF specialist if your child

- $\quad$ is not gaining weight

- $\quad$ has loose stools, very bad gassiness or constipation that last more than 2 weeks

- $\quad$ has very bad stomach aches

- $\quad$ has coughing or wheezing that last more than 2 weeks

If your CF specialist sees your child in an office where there a people with $\mathrm{CF}$, they may take special precautions to be sure that your child does not is not exposed to the germ called Pseudomonas. It is important to know that Pseudomonas is everywhere, and even healthy babies might have Pseudomonas.

What can we do to keep our child healthy?-As is true for all children, people with CRMS should not be exposed to cigarette smoke. All children who are over 6 months of age should receive yearly influenza vaccine. 


\section{List of abbreviations:}

$\begin{array}{ll}\text { CF } & \text { cystic fibrosis } \\ \text { CFTR } & \text { cystic fibrosis transmembrane conductance regulator } \\ \text { NBS } & \text { newborn screening } \\ \text { CRMS } & \text { CFTR-related metabolic syndrome } \\ \text { ICD } & \text { International Classification of Diseases } \\ \text { PCP } & \text { primary care provider } \\ \text { PI } & \text { pancreatic insufficient } \\ \text { PS } & \text { pancreatic sufficient } \\ \text { RSV } & \text { respiratory syncitial virus }\end{array}$

\section{References}

1. Borowitz D RK, Rosenfeld M, Davis S, Sabadosa K, Spear S, Michel S, White TB, Marshall B, Accurso FA. Cystic Fibrosis Foundation Evidence-Based Guidelines for Management of Infants with Cystic Fibrosis. Journal of Pediatrics 2009; current supplement.

2. Farrell PM, Rosenstein BJ, White TB, Accurso FJ, Castellani C, Cutting GR, et al. Guidelines for diagnosis of cystic fibrosis in newborns through older adults: Cystic Fibrosis Foundation consensus report. J Pediatr 2008;153(2):S4-S14. [PubMed: 18639722]

3. Castellani C, Cuppens H, Macek M, Jr., Cassiman JJ, Kerem E, Durie P, et al. Consensus on the use and interpretation of cystic fibrosis mutation analysis in clinical practice. J Cyst Fibros 2008;7(3): 179-96. [PubMed: 18456578]

4. Miller J, Petrie J. Development of practice guidelines. Lancet 2000;355(9198):82- [PubMed: 10675160]

5. http://is.njit.edu/pubs/delphibook/index.html\#toc

6. Scotet V, Audrezet MP, Roussey M, Rault G, Dirou-Prigent A, Journel H, et al. Immunoreactive trypsin/DNA newborn screening for cystic fibrosis: should the R117H variant be included in CFTR mutation panels? Pediatrics 2006;118(5):e1523-9. [PubMed: 17015492]

7. Massie RJ, Poplawski N, Wilcken B, Goldblatt J, Byrnes C, Robertson C. Intron-8 polythymidine sequence in Australasian individuals with CF mutations R117H and R117C. Eur Respir J 2001;17(6):1195-200. [PubMed: 11491164]

8. Peckham D, Conway SP, Morton A, Jones A, Webb K. Delayed diagnosis of cystic fibrosis associated with $\mathrm{R} 117 \mathrm{H}$ on a background of $7 \mathrm{~T}$ polythymidine tract at intron 8 . J Cyst Fibros 2006;5(1):63-5. [PubMed: 16266832]

9. Mussaffi H, Prais D, Mei-Zahav M, Blau H. Cystic fibrosis mutations with widely variable phenotype: the D1152H example. Pediatr Pulmonol 2006;41(3):250-4. [PubMed: 16429425]

10. Noone PG, Pue CA, Zhou Z, Friedman KJ, Wakeling EL, Ganeshananthan M, et al. Lung disease associated with the IVS8 5T allele of the CFTR gene. Am J Respir Crit Care Med 2000;162(5): 1919-24. [PubMed: 11069835]

11. Cuppens H, Lin W, Jaspers M, Costes B, Teng H, Vankeerberghen A, et al. Polyvariant mutant cystic fibrosis transmembrane conductance regulator genes. The polymorphic (Tg)m locus explains the partial penetrance of the T5 polymorphism as a disease mutation. J Clin Invest 1998;101(2):487-96. [PubMed: 9435322]

12. Groman JD, Hefferon TW, Casals T, Bassas L, Estivill X, Des Georges M, et al. Variation in a repeat sequence determines whether a common variant of the cystic fibrosis transmembrane 
conductance regulator gene is pathogenic or benign. Am J Hum Genet 2004;74(1):176-9. [PubMed: 14685937]

13. Sims EJ, Clark A, McCormick J, Mehta G, Connett G, Mehta A. Cystic fibrosis diagnosed after 2 months of age leads to worse outcomes and requires more therapy. Pediatrics 2007;119(1):19-28. [PubMed: 17200267]

14. Farrell PM. The meaning of "early" diagnosis in a new era of cystic fibrosis care. Pediatrics 2007;119(1):156-7. [PubMed: 17200282]

15. O’Sullivan BP, Zwerdling RG, Dorkin HL, Comeau AM, Parad R. Early pulmonary manifestation of cystic fibrosis in children with the DeltaF508/R117H-7T genotype. Pediatrics 2006;118(3): 1260-5. [PubMed: 16951024]

16. Saiman L, Siegel J. Infection control recommendations for patients with cystic fibrosis: microbiology, important pathogens, and infection control practices to prevent patient-to-patient transmission. Infect Control Hosp Epidemiol 2003;24(5 Suppl):S6-52. [PubMed: 12789902]

17. Mayell SJ, Munck A, Craig JV, Sermet I, Brownlee KG, Schwarz MJ, et al. A European consensus for the evaluation and management of infants with an equivocal diagnosis following newborn screening for cystic fibrosis. J Cyst Fibros 2009;8(1):71-8. [PubMed: 18957277]

18. Lording A, McGaw J, Dalton A, Beal G, Everard M, Taylor CJ. Pulmonary infection in mild variant cystic fibrosis: implications for care. J Cyst Fibros 2006;5(2):101-4. [PubMed: 16426904]

19. Van Ewijk BE, Wolfs TF, Aerts PC, Van Kessel KP, Fleer A, Kimpen JL, et al. RSV mediates Pseudomonas aeruginosa binding to cystic fibrosis and normal epithelial cells. Pediatr Res 2007;61(4):398-403. [PubMed: 17515861]

20. O'Sullivan BP CE, Carlson D, Parad R, Comeau AM. Oropharyngeal flora in non-CF infants. Pediatric Pulmonology 2007;Suppl 30:323.

21. Kopelman H, Corey M, Gaskin K, Durie P, Weizman Z, Forstner G. Impaired chloride secretion, as well as bicarbonate secretion, underlies the fluid secretory defect in the cystic fibrosis pancreas. Gastroenterology 1988;95(2):349-55. [PubMed: 3391365]

22. Beharry S, Ellis L, Corey M, Marcon M, Durie P. How useful is fecal pancreatic elastase 1 as a marker of exocrine pancreatic disease? J Pediatr 2002;141(1):84-90. [PubMed: 12091856]

23. Chillon M, Casals T, Mercier B, Bassas L, Lissens W, Silber S, et al. Mutations in the cystic fibrosis gene in patients with congenital absence of the vas deferens. N Engl J Med 1995;332(22): 1475-80. [PubMed: 7739684]

24. Mak V, Zielenski J, Tsui LC, Durie P, Zini A, Martin S, et al. Proportion of cystic fibrosis gene mutations not detected by routine testing in men with obstructive azoospermia. JAMA 1999;281(23):2217-24. [PubMed: 10376575] 


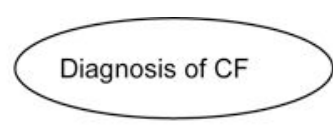

iagnosis of $\mathrm{CF}$

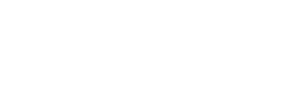

DNA analysis using multi-mutation method Ancillary tests
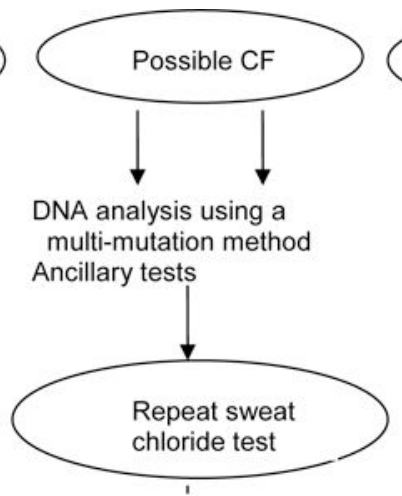

Individuals with one CF-causing mutation, no mutation on the other allele and an intermediate sweat chloride are presumed to be CF carriers.

Individuals with intermediate sweat chloride and no identified mutations may have other disease states associated with elevated sweat chloride (See Table II in: LeGrys VA. Sweat testing for the diagnosis of cystic fibrosis: practical considerations. J Pediatr 1996; 129:892-897 and www.hopkinsmedicine.org/dnadiagnostic/CFlikeBENaC.htm

\section{Figure 1:}

Individuals who have CFTR-Related Metabolic Syndrome; based on the Figure from Farrell et al (2) 
$\mathrm{X}=$ do at this visit

$\mathrm{C}=$ consider

\begin{tabular}{|c|c|c|c|c|}
\hline AGE AT VISIT $\rightarrow$ & $\begin{array}{c}\text { By } 2 \\
\text { months } \\
\text { of age }\end{array}$ & $\begin{array}{c}6 \\
\text { months } \\
\text { of age }\end{array}$ & $\begin{array}{l}\text { Age 1 } \\
\text { and } 2 \\
\text { years }\end{array}$ & Annually \\
\hline \multicolumn{5}{|l|}{ INTERVENTIONS $\downarrow$} \\
\hline \multicolumn{5}{|l|}{ CARE ISSUES } \\
\hline $\begin{array}{l}\text { Discuss diagnosis of CFTR-related } \\
\text { metabolic syndrome }\end{array}$ & $\mathrm{X}$ & $\mathrm{C}$ & $\mathrm{C}$ & $\mathrm{C}$ \\
\hline Discuss diagnosis with PCP & $\mathrm{X}$ & $\mathrm{C}$ & $\mathrm{C}$ & $\mathrm{C}$ \\
\hline Measure weight, length, OFC & $\mathrm{X}$ & $\mathrm{X}$ & $\mathrm{X}$ & $\mathbf{X}$ \\
\hline Vital signs and physical examination & $\mathrm{X}$ & $\mathrm{X}$ & $\mathrm{X}$ & $\mathbf{X}$ \\
\hline Influenza vaccine & & $\mathrm{X}$ & $\mathrm{X}$ & $\mathbf{X}$ \\
\hline \multicolumn{5}{|l|}{ DIAGNOSTIC TESTING } \\
\hline Sweat test & $X^{*}$ & $\mathrm{X}$ & & \\
\hline Fecal elastase & $\mathrm{X}$ & $\mathrm{C}$ & $\mathrm{C}$ & $\mathrm{C}$ \\
\hline Respiratory culture $^{2}$ & $\mathrm{X}$ & $\mathrm{X}$ & $\mathrm{X}$ & $\mathbf{X}$ \\
\hline Blood tests (see manuscript text) & $\mathrm{C}$ & $\mathrm{C}$ & $\mathrm{C}$ & $\mathrm{C}$ \\
\hline Chest x-ray ${ }^{3}$ & $\mathrm{C}$ & $\mathrm{C}$ & $\mathrm{C}$ & $\mathrm{C}$ \\
\hline Abdominal $\mathrm{x}$-ray & $\mathrm{C}$ & $\mathrm{C}$ & $\mathrm{C}$ & $\mathrm{C}$ \\
\hline Spirometry (by standard adult methods) ${ }^{4}$ & & & & $\mathbf{X}$ \\
\hline \multicolumn{5}{|l|}{ EDUCATION } \\
\hline Infection Control & $\mathrm{X}$ & $\mathrm{C}$ & $\mathrm{C}$ & $\mathrm{C}$ \\
\hline Tobacco smoke exposure avoidance & $\mathrm{X}$ & $\mathrm{X}$ & $\mathrm{X}$ & $\mathbf{X}$ \\
\hline Genetic counseling & $\mathbf{X}$ & $\mathrm{C}$ & C & C \\
\hline
\end{tabular}

* Recommend waiting until 2 months of age before performing the repeat sweat test.

1. Repeat fecal elastase only if individuals are symptomatic

2. Treat patients per CF protocol if Pseudomonas positive; acknowledge that healthy infants can be intermittently Pseudomonas positive

3. Obtain $\mathrm{x}$-rays only if patients are symptomatic

4. Start monitoring when individuals are old enough to perform standard spirometry

Figure 2:

Management of Individuals with a Diagnosis of CFTR-Related Metabolic Syndrome 
Table 1:

Examples of CFTR mutations with regard to their clinical consequences

\begin{tabular}{|c|c|}
\hline Mutation Group & Examples \\
\hline A. "CF-causing" 1 & 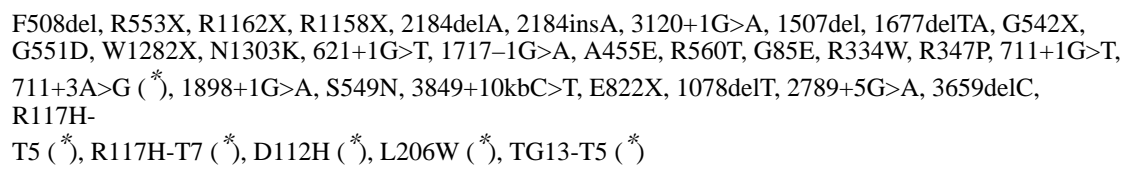 \\
\hline $\begin{array}{l}\text { B. "CFTR-related disorders } \\
\text { associated" }\end{array}$ & $\begin{array}{l}\left.\left.\left.\left.\text { R117H-7T ( }{ }^{*}\right), \text { TG12-T5 ( }{ }^{*}\right), \text { R117H-T5 ( }{ }^{*}\right), \text { D1152H }\left(^{*}\right), \text { TG13-T5 ( }{ }^{*}\right), \text { S997F, R297Q }\left(^{*}\right), \text { L997F, } \\
\text { M9521, D565G }\left(^{*}\right), \text { G576A }\left(^{*}\right), \text { TG11-T5 }\left(^{* *}\right), \text { R668C-G576A-D443Y, R74W-D1270N }\end{array}$ \\
\hline C. "No clinical consequences" & $\begin{array}{l}\text { I148T, R75Q, 875+40A/G, M470V, E52E, T854T, P1290P, 2752- } \\
\text { 15G/C, I807M, 1521F, F508C, I506V, TG11-T5 (**) }\end{array}$ \\
\hline $\begin{array}{l}\text { D. "Unknown or uncertain } \\
\text { clinical relevance" }\end{array}$ & Many missense mutations $\left({ }^{* * *}\right)$ \\
\hline
\end{tabular}

${ }^{1}$ Note that the Guidelines for Diagnosis of CF in Newborns through Older Adults ${ }^{2}$ lists a panel of 23 CF-causing mutations in this category, revised from the mutation panel for population screening for CF developed by the American College of Medical Genetics.

${ }^{(*)}$ Mutations which may belong either to Group A or Group B

${ }^{(* *)}$ Mutations which may belong either to Group B or Group C

${ }^{(* * *)}$ Certain common sequence (missense) variants with subclinical molecular consequences (e.g. M470V) may co-segregate on the same chromosome and exert more potent, cumulative phenotypic effect. 
Table 2:

\section{Delphi Questions and Level of Consensus}

Italicized statements never reached the level of consensus to become recommendations

\begin{tabular}{|c|c|}
\hline Statement Number & Statement \\
\hline 1 & A genetic counselor should be present, if possible, in the discussion of genetic findings in individuals with CRMS. \\
\hline 2 & The CF Specialist should communicate directly with the primary care provider to explain the implications of CRMS. \\
\hline 3 & $\begin{array}{l}\text { The PCP should contact the CF specialist if a individual with CRMS is not gaining weight, has loose stools and/or } \\
\text { flatus, has abdominal pain or has respiratory symptoms such as cough or wheezing that do not resolve in } 2 \text { weeks. }\end{array}$ \\
\hline 4 & A clinical assessment by a CF specialist should be performed by 2 months of age. \\
\hline 5 & $\begin{array}{l}\text { To prevent possible cross-infection, infants with CRMS should be assessed in a clinic adherent to CF Foundation } \\
\text { guidelines suggested for CF patients, or in a separate clinic area from CF patients as local conditions allow. }\end{array}$ \\
\hline 6 & CF Center ancillary personnel may need to see individuals with CRMS; this can be scheduled on an as-needed basis. \\
\hline 7 & $\begin{array}{l}\text { The following should be done at the time of the initial assessment: comprehensive history and physical including } \\
\text { accurate weight and height measurements, an objective measure of pancreatic function, and an oropharyngeal culture. }\end{array}$ \\
\hline 8 & In a individual with CRMS a chest $\mathrm{x}$-ray should be done if respiratory symptoms are present. \\
\hline 9 & $\begin{array}{l}\text { The following laboratory tests may be done at the time of the initial assessment of an individual with CRMS if they } \\
\text { have not been done recently: complete blood count with differential. }\end{array}$ \\
\hline 10 & $\begin{array}{l}\text { The following laboratory tests may be considered at the time of the initial assessment of an individual with CRMS if } \\
\text { they have not been done recently: Liver function tests (AST, ALT, GGT, bilirubin). }\end{array}$ \\
\hline 11 & $\begin{array}{l}\text { The following laboratory tests may be considered at the time of the initial assessment of an individual with CRMS if } \\
\text { they have not been done recently: albumin. }\end{array}$ \\
\hline 12 & $\begin{array}{l}\text { The following laboratory tests may be considered at the time of the initial assessment of an individual with CRMS if } \\
\text { they have not been done recently: vitamin } A \text { and E levels. }\end{array}$ \\
\hline 13 & $\begin{array}{l}\text { Patients should be treated per CF protocol if Pseudomonas is found on oropharyngeal swab, even if a definitive } \\
\text { diagnosis of CF has not been established. (Note: wording changed from "should" to "may" based on comments from } \\
\text { public posting) }\end{array}$ \\
\hline 14 & $\begin{array}{l}\text { Asymptomatic individuals with CRMS should be seen at least twice during the first year of life and once a year } \\
\text { thereafter. Some asymptomatic individuals may need more visits, depending on the comfort level of the family. }\end{array}$ \\
\hline 15 & Oropharyngeal cultures should be obtained at every visit. \\
\hline 16 & $\begin{array}{l}\text { 1/8 tsp table salt per day may be considered for individuals with CRMS who have sweat chloride levels at the higher } \\
\text { end of the intermediate range. }\end{array}$ \\
\hline 17 & $\begin{array}{l}\text { Multivitamins designed for persons who have CF should be prescribed to individuals with CRMS if there is laboratory } \\
\text { evidence of vitamin A or vitamin E deficiency. (Note: recommendation expanded to include the need for evaluation of } \\
\text { malabsorption if vitamin deficiency is noted based on comments from public posting) }\end{array}$ \\
\hline 18 & $\begin{array}{l}\text { In the absence of clinical or radiologic evidence of lung disease, routine airway clearance therapy should not be } \\
\text { prescribed for individuals with CRMS. }\end{array}$ \\
\hline 19 & Individuals with CRMS should not be exposed to cigarette smoke. \\
\hline 20 & Individuals with CRMS should receive yearly influenza vaccine. \\
\hline 21 & $\begin{array}{l}\text { Pancreatic sufficient individuals with poor weight gain, loose stools and/or flatus, and/or abdominal pain should have an } \\
\text { objective measure of pancreatic functional status to rule out conversion to pancreatic insufficiency. }\end{array}$ \\
\hline 22 & $\begin{array}{l}\text { An abdominal x-ray should be considered in pancreatic sufficient individuals with CRMS with poor weight gain, loose } \\
\text { stools and/or flatus, and/or abdominal pain to rule out constipation. }\end{array}$ \\
\hline 23 & $\begin{array}{l}\text { Airway clearance therapy may be considered if individuals with CRMS have recurrent or prolonged respiratory } \\
\text { symptoms or have chest X-ray findings suggestive of lower airway disease. }\end{array}$ \\
\hline
\end{tabular}




\begin{tabular}{|c|c|c|c|c|c|c|}
\hline \multicolumn{7}{|c|}{ Agreement Levels for the First Two Rounds* } \\
\hline & \multicolumn{3}{|c|}{ Round 1} & \multicolumn{3}{|c|}{ Round 2} \\
\hline Statement number & $\begin{array}{c}\text { Consensus (\%) } \\
\text { Scores between } 7 \text { and } 9\end{array}$ & Range & $\underset{\text { (median) }}{\text { Mean }}$ & $\begin{array}{c}\text { Consensus (\%) } \\
\text { Scores between } 7 \text { and } 9\end{array}$ & Range & $\begin{array}{c}\text { Mean } \\
\text { (median) }\end{array}$ \\
\hline 1 & 66 & $1-9$ & $7(7)$ & 83 & $4-9$ & $8(9)$ \\
\hline 2 & 94 & $4-9$ & $9(9)$ & & & \\
\hline 3 & $89^{* * *}$ & $4-9$ & $9(9)$ & 94 & $6-9$ & $9(9)$ \\
\hline 4 & 94 & $1-9$ & & & & \\
\hline 5 & 83 & $4-9$ & $8(9)$ & & & \\
\hline 6 & 100 & $7-9$ & $8(9)$ & & & \\
\hline 7 & 94 & $6-9$ & $9(9)$ & & & \\
\hline 8 & $61^{* * * *}$ & $1-9$ & $7(8)$ & 94 & $6-9$ & $9(9)$ \\
\hline 9 & 78 & $3-9$ & $8(9)$ & 76 & $5-9$ & $7(8)$ \\
\hline 10 & 78 & $0-9$ & $7(8)$ & 65 & $0-9$ & $7(7)$ \\
\hline 11 & 72 & $3-9$ & $7(8)$ & 76 & $0-9$ & $8(8)$ \\
\hline 12 & 78 & $1-9$ & $7(8)$ & 76 & $0-9$ & $8(8)$ \\
\hline 13 & 83 & $2-9$ & $8(9)$ & & & \\
\hline 14 & 50 & $0-9$ & $5(6)$ & 94 & $5-9$ & $8(8)$ \\
\hline 15 & 94 & $6-9$ & $8(9)$ & & & \\
\hline 16 & $67^{* * * *}$ & $0-9$ & $7(8)$ & 70 & $0-9$ & $7(8)$ \\
\hline 17 & $72^{* * * *}$ & $2-9$ & $7(8)$ & 94 & $6-9$ & $8(9)$ \\
\hline 18 & $78^{* * * *}$ & $3-8$ & $7(8)$ & 94 & $5-9$ & $8(9)$ \\
\hline 19 & 100 & 9 & $9(9)$ & & & \\
\hline 20 & 94 & $6-9$ & $9(9)$ & & & \\
\hline 21 & 100 & $7-9$ & $9(9)$ & & & \\
\hline 22 & 61 & $0-9$ & $7(8)$ & 82 & $3-9$ & $8(9)$ \\
\hline 23 & 50 & $1-9$ & $6(6)$ & 88 & $4-9$ & $9(9)$ \\
\hline
\end{tabular}

The wording of some questions was modified between Rounds 1 and 2 based on feedback from the participants; a second round was not done if > $80 \%$ consensus was achieved on Round 1 and if there was not a significant change in wording

In this question, the interval for intervention was changed from 1 week to 2 weeks between Round 1 and Round 2

For these questions, the wording changed from Round 1 to Round 2 to emphasize intervention if patients were symptomatic or had a test result that informed the action 
Table 3.

Medical Issues to Discuss with the Family at the Time of Identification of CFTR-Related Metabolic Syndrome

- Assess emotional and educational status of the family

- Explain basic genetic concepts

$\bigcirc$ be sure that formal genetic counseling has been provided or is scheduled

- Provide a general description of CF symptoms and what causes them

$\bigcirc$ Explain that there is a lot of incorrect and outdated information about CF (e.g. from friends and family, in books, and on the web)

- Explain why we think the infant likely does not have $\mathrm{CF}$

- Explain how we know the infant has CRMS

What was the process that led to this diagnosis

$O$ What does it mean if a child has CRMS

- Convey the news that we are uncertain about the prognosis:

$\bigcirc$ We expect this child to lead a full life

Some, but not all individuals with CRMS may develop symptoms that should be treated

- Some individuals with CRMS are at high risk for male infertility

This is why we need to establish the current baseline and follow this child in our clinic

We may not have all the answers right now, but new information becomes available all the time

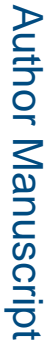

- If individuals with CRMS cannot be seen separately from patients with CF, discuss infection control

- Describe the monitoring plan, how to contact the CF Care Center with questions or concerns; schedule the next visit before the family leaves 
Table 4:

Issues about CFTR-related Metabolic Syndrome for Primary Care Providers

- Phenotype is more important than genotype

$>$ Many infants with CRMS will continue to be healthy and will thrive

$>$ Males with CRMS may be at higher risk of infertility

- Early symptoms can be subtle; open communication with the $\mathrm{CF}$ specialist is important if there are concerning symptoms such as:

Lack of weight gain or unresolved acute weight loss

Persistent loose stools or excessive flatus

$\bigcirc$ Abdominal pain

$\bigcirc$ Coughing or wheezing

$>$ Contact the CF specialist if these symptoms persist $>2$ weeks

- If individuals with CRMS develop symptoms they may benefit from current and new treatments

- The CF Center is a resource for:

$>$ Medical advice

Psychosocial support for families who have difficulty coping with the uncertainty of this diagnosis

Information about new developments as they become available 